\title{
Effects of the Different Modification Treatment Liners on Tribological Properties of Self-Lubricating Spherical Plain Bearings and the Bonding Property of Liners
}

\author{
Ming Qiu ${ }^{1, a}$, Xia Liang ${ }^{2, b}$, Yingchun $\mathrm{Li}^{3, \mathrm{c}}$, Xiaoxu Pang ${ }^{4, \mathrm{~d}}$ \\ ${ }^{1,2,3,4}$ School of Mechatronics Engineering, Henan University of Science and \\ Technology, Luoyang 471003, Henan Province, PR China

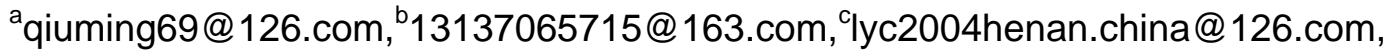 \\ dpxx8308@163.com
}

Keywords: spherical plain bearing; modification; bonding property; friction and wear

\begin{abstract}
PTFE/aramid woven fiber liners were modified by acrylamide, phosphate ester coupling agent, respectively. The effects of the modified liner on the friction coefficient, the wear loss and the friction temperature of self-lubricating spherical plain bearings were investigated by using self-made compound swinging type friction and wear testing machine. With Instron 5944 electronic universal material testing machine, the bonding property was analyzed.The results show that the bonding property of the modified liners have a greater degree of improvement and the friction coefficient, the wear loss and the friction temperature of the bearing were all decreased. In the three kinds of liners, the bonding properties and tribological properties of spherical plain bearing with modified by acrylamide liners were the best.
\end{abstract}

\section{Introduction}

Self-lubricating spherical plain bearings are widely used in practical engineering because of their advantages of the high load carrying and maintenance-free capacities[1,2]. With the increased requirements in the aerospace, railway machinery and other fields, the higher safety and reliability of the bearing are required[3,4] .To realize the capacities, a layer of self-lubricating materials is bonded on the internal spherical surface of the outer ring of the bearing[5,6].The wear failure is the main failure mode of the self-lubricating spherical plain bearing[7] ,the friction and wear properties of PTFE/aramid fiber composite were studied by some scholars, but little research has been done on the friction and wear properties of the modified PTFE/aramid fiber hybrid woven liner. Some studies have shown that the surface treatment of the liner of self-lubricating spherical plain bearings can improve the friction and wear properties[8].PTFE/aramid woven fiber liner has excellent self lubrication and wear resistance properties, but the aramid fiber surface as the adhesive surface of the liner is smooth, the steric effect of the benzene ring is large, it lacks active groups, which leads to a poor invasion between the liner and the adhesive interface, the adhesion is poor, so as to the tribological performance of self lubricating liner is affected. The modified treatment liner was modified by the strong grafting activity of large molecular chain on the fiber bundle, which can optimize the interfacial properties of aramid fiber surface, so the physical adhesion between the fiber surface and the adhesive were improved[9].In this paper, the peel strength and tribological properties of different types of bearings were studied, and the effects of different modification treatment liners on the bonding properties and tribological properties of the self lubricating spherical plain bearing were discussed.

\section{Experiment}

\section{Samples preparation.}

The model of the self-lubricating spherical plain bearings used in the test is GE20ET-2RS,the basic structure of bearing is shown in Fig.1 the hybrid woven liners were weaved with PTFE and aramid, of which the surfaces being rich in aramid fibers were used as the adhesive surfaces .the liner 
was modified, detailed plan is shown in table 1.then the modified and unmodified liners were bonded to the inner spherical surface of the outer ring with adhesive, after the inner rings were installed the outer rings, the spherical plain bearings were cured in the oven.

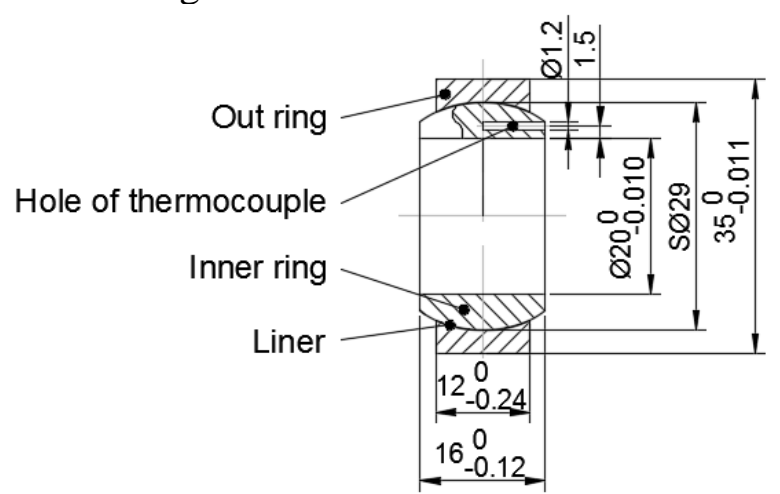

Fig.1 The basic structure of the self-lubricating spherical plain bearing

Table 1 friction and wear test material of spherical plain bearing

\begin{tabular}{cccc}
\hline spherical plain bearing & $\begin{array}{c}\text { The materials of inner and } \\
\text { outer ring }\end{array}$ & $\begin{array}{c}\text { The materials of } \\
\text { liners }\end{array}$ & Liners pretreatment \\
\hline 1 & GCr15 & PTFE//Kevlar & Untreated \\
3 & GCr15 & PTFE/Kevlar & AcrylamideTreated \\
GCr15 & PTFE//Kevlar & Phosphate ester coupling agent \\
\end{tabular}

The liner adopts three treatment options,the specific process is as follows:

Acrylamide treated[10]: the liner is placed in the prepared acrylamide solution to be immersed for $3 \mathrm{~min}$ at room temperature, and then dried in the oven at temperature of $110^{\circ} \mathrm{C}$ for $30 \mathrm{~min}$,so that the surface reaction occurs.

Phosphate ester coupling agent treated[11]: the liner was impregnated in the alcohol solution which was prepared for $15 \mathrm{~min}$, and then dried in the oven at temperature of $95^{\circ} \mathrm{C}$ for $15 \mathrm{~min}$.

\section{Test method.}

\section{1) Peel test.}

The peel strength is one of the quantitative evaluation parameters of the bonding quality of the liner. The bearing is clamped to the instron5944 type electronic universal material testing machine by using the self made spherical plain bearing peel fixture, the liner was peeled off from the internal surface of the outer ring.

The calculation of peel strength is quadrature of the peeling curve by using origin software, the experiment was repeated three times and taken average peel strength of each test, the specific experimental formula is as follows:

$$
\sigma=C \times \frac{S}{L B}
$$

$\sigma$ peel strength, $\mathrm{N} / \mathrm{mm}$; $C$ The load represented by the unit height on the stripping curve, $\mathrm{N} / \mathrm{mm} ;{ }^{S}$ Quadrature of the stripping curve, $L$ peel length of liner, mm, $B$ Width of peeling sample, $\mathrm{mm}$.

According to the related standard of SAE-AS81820 and combined with actual conditions, the peel angle of the test is $140^{\circ} \pm 40^{\circ}$; the peel rate is $19 \mathrm{~mm} / \mathrm{min}$ and collection interval is $2 \mathrm{~ms}$.It should make an adhesion test for the liner before peeling off it to ensure the liner cling by $90 \%$.We should taking a firmness test for the liner after peeling off it.

\section{2) Friction and wear test.}

The experiment was carried out on the self-made compound swing friction and wear test machine, as shown in Fig.2. According to the relevant standard of SAE-AS81819 and SAE-AS81820 and 
combined with the actual conditions to work out the test plan. The test was carried out at room temperature, the contact pressure of the bearing was $15 \mathrm{kN}$, the swing frequency is $1.5,2,2.5,3 \mathrm{~Hz}$, and bearing rotation direction of the swing angle is about $\pm 10^{\circ}$, and hold the static pressure for $20 \mathrm{~min}$,and then the rotation swing test was carried out for 25000 times. The measurement of the friction coefficient is obtained by measuring the torque value measured by torque sensor and referring to the definition of the friction coefficient. The wear loss was characterized by the displacement of radial line between inner and outer rings measured by displacement sensor. The frictional temperature is measured by the thermo couple sensor which is inserted into a $\Phi 1.2 \mathrm{~mm}$ hole on the end face of bearing inner ring. The test is repeated three times and taken average friction coefficient, wear loss and friction temperature rise of each test.

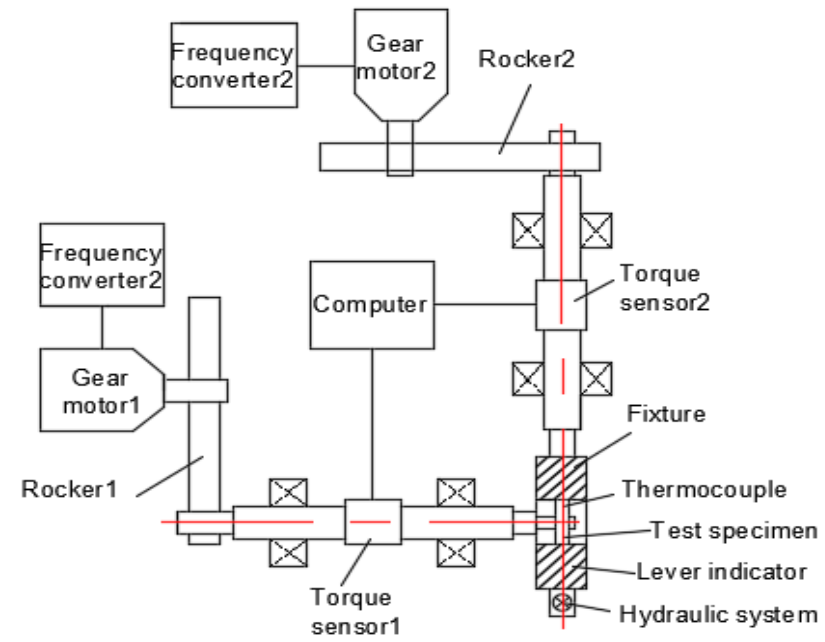

Fig 2. Schematic diagram of testing machine for tribology performance of spherical plain bearing

\section{Results and discussion}

Influence of modified liner on bonding property of self-lubricating spherical plain bearing.

The repeated experiments were performed on the samples for 7 times, the peel strength of seven times repeated experiments were taken average, as shown in table 2, the bonding strength of the liner modified with acrylamide increase maximum; phosphate coupling agent modification processing is relatively low.

Table 2 Results of peel strength test

\begin{tabular}{cccc}
\hline sample & peel strength/(N.mm) & Integrity checking & Reliability \\
& & & checking \\
\hline Untreated & 0.7858 & passed & passed \\
Acrylamide Treated & 0.94262 & passed & passed \\
Phosphate ester coupling agent & & passed & passed \\
Treated & 0.825 & & \\
\hline
\end{tabular}




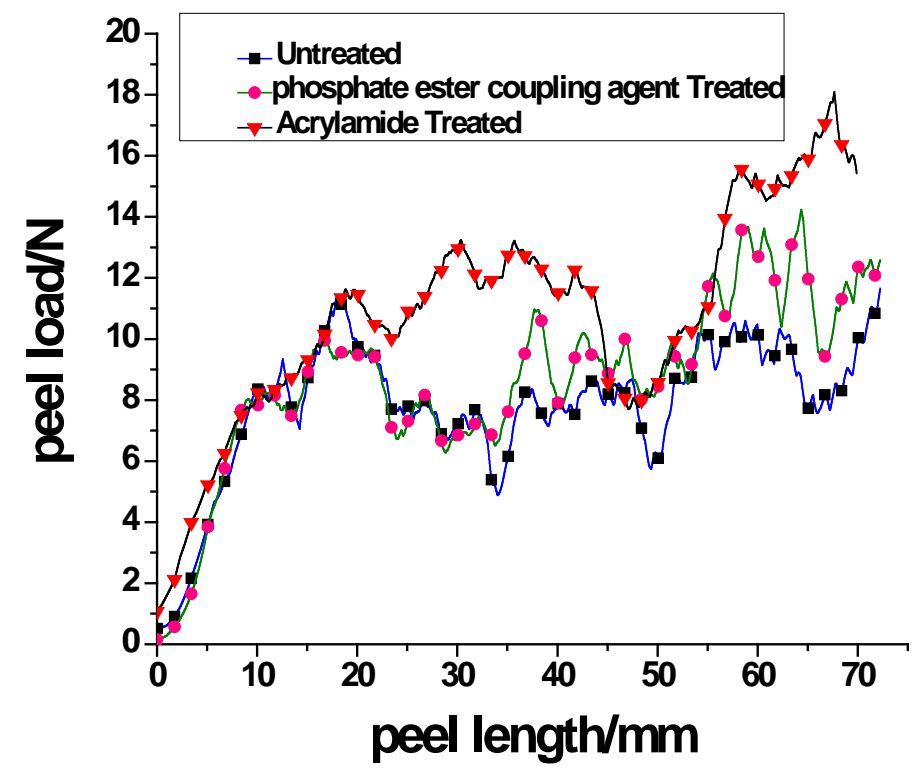

Fig.3.the peel strength curve of the spherical plain bearings with the liners modified and unmodified



(a)

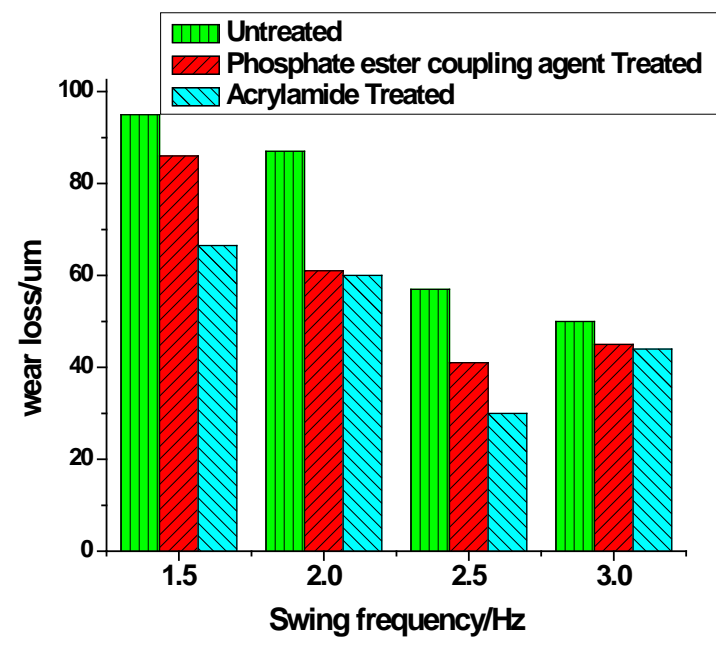

(b)

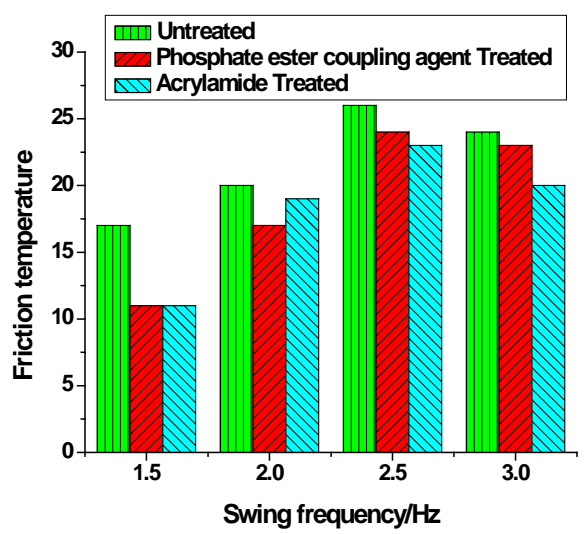

(c)

Fig.4.the tribological properties of the spherical plain bearing with the liners modified and unmodified along with the swing frequency:(a) friction coefficient (b) wear loss (c) friction temperature 
Fig 3 shows the peeling curve of liner of the spherical plain bearing before and after modification,the peel load of bearings with modified liners all increased compared with that with unmodified liners. the peel load of untreated liner is relatively low, the maximum peel load is about $13 \mathrm{KN}$,when the peel length is $12 \mathrm{~mm}$ and $34 \mathrm{~mm}$,the peel strength has obvious mutation,it shows that the bonding strength and the bonding behaviors poor,it is easy to cause the self lubrication spherical plain bearing to fall off due to the poor adhesion between the liner and the matrix in the course of using, and the friction coefficient is increased. The peel load of the bearing was significantly increased after the treatment with acrylamide,and it is larger than the peel load of the untreated bearing,overall the bonding performance is enhanced. the adhesive strength and the bonding behavior of the bearing is relatively improved. After the treatment of phosphate coupling agent, the peel strength was improved,which showed that the bonding behavior of the liner was improved after the treatment. In general, the bonding properties of self-lubricating spherical plain bearing treated by acrylamide were the best.

\section{Influence of modified liner on tribological properties of self-lubricating spherical plain bearing.}

Seen from Fig4, the friction coefficient, the wear loss and the friction temperature of spherical plain bearing with modified liners all decreased. As is shown in Fig 4(a), with the increase of the swing frequency, the friction coefficient of the three types of bearings is presented a decreasing trend, from the slope, reduction rate of the friction coefficient decreases with the increase of the swing frequency, at $1.5 \sim 2.5 \mathrm{~Hz}$, the friction coefficient of the bearing modified by acrylamide is the lowest, followed by the treatment of phosphate coupling agent bearing, the friction coefficient of untreated bearing is largest. After $2.5 \mathrm{~Hz}$, the friction coefficient of the three kinds of bearings is reduced, because frictional heating changed PTFE fiber / aramid fiber composite fabric surface microstructure and tribological properties when frequency is too large.

As is shown in Fig 4(b),the wear loss of the liners of the modified bearings is lower than the wear loss of the untreated bearing,In general,the wear loss of the bearing after acrylamide treatment is the lowest,phosphate coupling agent treatment was followed by,and the wear loss of the bearing untreated is the largest,.Mainly because the liner after treatment promote the formation of PTFE transfer film,the wear loss is reduced,the tribological properties are improved.

Fig 4(c) shows that the friction temperature of the bearings with modified liners is lower than that of the untreated one,the maximum value of friction temperature rise occurs when the swing frequency is $2.5 \mathrm{~Hz}$,it is mainly because the sliding speed of the dual surface is increased and the friction coefficient is decreased with the increase of the bearing swing frequency,resulting in reducing the heat generated by friction,thus inhibiting the bearing temperature rise.

\section{Summary}

(1)The peel strength of the spherical plain bearing was improved after modified treatment. Among them, the peel strength was improved most by the modification of acrylamide. It is showed that the bonding property of the fiber surface is improved by the modification of the liner.

(2)Under the different swing frequencies, the friction coefficient, the wear loss and the friction temperature of the bearing after modification of liner is lower than that of the untreated bearing, after the liner was modified by acrylamide, the decrease amplitude of the friction coefficient and the wear loss of the bearing are relatively large, the tribological properties of the untreated bearing were the worst. The tribological properties of the self lubricating spherical plain bearing were improved after the liner was modified.

(3)The bonding properties and tribological properties of the liners were analyzed before and after modification. In terms of bonding properties, the peel strength of the bearing is the highest after the modification of the liner by acrylamide, the peel strength of the untreated bearing is the lowest .in terms of tribological properties, under the same working condition, the wear loss and the friction coefficient of the bearing with modified liner by acrylamide are the lowest, that of untreated bearing 
are the largest, it is showed that improving the bonding strength of the liner is helpful to improve the tribological performance of the self lubricating spherical plain bearing when swing frequency is low.

\section{Acknowledgements}

The authors are grateful to financial support from the National Natural Science Foundation of China (Grant No. 51275155), Outstanding Talent Fund Projects of Henan Province (No. 154200510013), and Innovation and Research Team of Science and Technology in Universities in Henan Province (No. 13IRTSTHN025).

\section{Reference}

[1] Xiang D.H., Wang C.D., Dong W.F. Structural Optimization and Friction and Wear Behavior of a Radial Spherical Plain Bearing. Tribology, 2004, 24 (6): 564-567.

[2] Zhou J., Zhu H.M., Zhou K. Self lubrication spherical plain bearing. Bearing, 2003 (7): 11-12.

[3] Zong C.L., Xie X., Zhou L., et al. Design principles of embedded self lubricating spherical plain bearing for hydraulic engineering. Bearing, 2004 (3): 8-9.

[4] Yang Y.l., Zu H., Huang H. Present situation and development of self lubricating spherical plain bearing. Bearing, 2009 (1): 58-61.

[5] Craig J.r. W D. Predicting spherical bearing life in airplane control system. Lubrication Engineering, 1962, 5(2): 25-29.

[6] Germaneau A, Peyruseigt F, Mistou S et al. 3D mechanical analysis of aeronautical plain bearings: validation of a finite element model from measurement of displacement fields by digital volume correlation and optical scanning tomography. Optics and Lasers in Engineering. 2010, 48(6): 676-683.

[7] Bai Y.X., Qiu M., Li Y.C. Joint bearing wear failure. Modern manufacturing engineering, 2012 (4): 138-142

[8] Briggs. Polymer surface analysis. Beijing: Chemical Industry Press, 2001.

[9] Liu X.J., Li T.S., Yao J.L., et al. Effect of surface treatment on the adhesion and friction properties of aramid fabric. Mechanical engineering materials, 2000, 24 (3): 38-42.

[10]Hu T.J., SJ. Fiber surface modification method, fiber products and uses: Patent 101368333A. 2009-2-18.

[11]Chen X.C., Zhang S., Xu G.Z., et al. Study on the properties of modified aramid fiber reinforced polypropylene composites. Plastic science and technology, 2011, 39 (4): 64-68. 